\title{
RSSI-based Rings Overlap Localization Algorithm in Wireless Sensor Network
}

\author{
Yuqiang Qin ${ }^{1, *}$ and Hui Ying ${ }^{2}$ \\ ${ }^{I}$ College of Economics and Management, Taiyuan University of Science and Technology, Taiyuan, Shanxi, 030024, P.R. \\ China; ${ }^{2}$ Taiyuan Normal University, Taiyuan, Shanxi, 030001, P.R. China
}

\begin{abstract}
This paper proposes a RSSI-based rings overlap localization algorithm of Environment Adaption(RROLA/EA) for large-scale wireless sensor network (WSN). Localization technology is one of the key technologies in most applications of wireless sensor network. Localization technology is composed of two phases: ranging phase and localization phase. RSSI-based ranging method is adopted in the ranging phase and rings overlap method is adopted in the localization phase. Firstly, through binary linear regression the parameters of signal attenuation are calibrated for a certain environment. Then, through differential error estimate method the inner radius and outer radius of rings are got respectively around beacon nodes and blind node. At last, the centroid of the rings overlapping region is taken as the location of blind node. Theoretical analyses and simulation results under different scenarios prove the correctness and validity of the localization algorithm.
\end{abstract}

Keywords: Binary linear regression, differential error estimate, rings overlap method, RSSI.

\section{INTRODUCTION}

Wireless sensor network is one of the three future hightech industries around the world. It has a wide range of applications in military, environmental monitoring, antiterrorism, health care, industrial control, smart home, business and other fields [1]. The network is an event-oriented monitoring network. For most applications, sensed dates without location information are pointless. Real-time acquisition of nodes' location information is one of the basic functions of wireless sensor network. Therefore, localization technology is one of the key technologies in most applications of wireless sensor network [2].

Localization technology is composed of two phases: ranging phase and localization phase. According to the difference of the ranging phase, the localization algorithm can be divided into two categories: range-based localization technology and range-free localization technology. Although the latter has lower hardware costs and lower power consumption, the expense of localization accuracy made it can be hardly applied in those projects with high localization accuracy requirement. On the contrary, the former is mainly used for the field of high-precision localization. The localization algorithm proposed in this paper is one kind of rangebased localization technology.

In ranging phase, there are four kinds of ranging technologies, that are RSSI, TOA, TDOA and AOA [3]. TDOA and AOA respectively use the difference of signal arrival times and signal arrival angles to calculate the distance between two nodes. These two kinds of ranging method have high ranging accuracy, but need extra hardware support. TOA ranging method uses the propagation delay time to compute the distance between two nodes. It has harsh demand on clock synchronization, and minimal clock drift can be converted into large ranging error. RSSI ranging principle is to convert propagation loss into distance based on the theoretical or empirical ranging model. RSSI ranging method is simple to operate, low-cost, and has been widely used.

A large number of RSSI-based localization algorithms have been developed in recent years. There're three major problems in those algorithms. Firstly, the channel attenuation index of the ranging model tends to take an experience value which is strongly subjective and seriously affects the localization accuracy. Secondly, the centroid of circular overlapping area was widely adopted as the blind node location. Thirdly, the ability to handle new nodes adds to network or old nodes fail off network was lacked [4].

To overcome the demerits of traditional RSSI-based localization algorithm, this paper proposes an environment adaption of rings overlap localization algorithm (EAROLA), which uses RSSI-based ranging method in the ranging phase and uses rings overlapping method in the localization phase [5].

\section{ALGORITHM DESCRIPTIONS}

\subsection{Description of Localization System}

In wireless sensor networks, the location known nodes are called beacon nodes while the location unknown nodes are called blind nodes [6]. The concept of slave beacon node was introduced, which refers to the nearest beacon node 
away from a blind node. That is, to a blind node, it can receive the largest RSSI value from its slave beacon node. The relationship between beacon nodes, blind node and slave beacon node is shown in Fig. (1). Firstly, the blind node M1 communicates with beacon nodes to determine the one-hop neighbor nodes and two-hop neighbors. The one-hop neighbor nodes are within the communication range of the blind node while the two-hop neighbor nodes are beyond the communication range and within two-hop communication range. Then, the neighbor node from which received the biggest RSSI value was confirmed as the slave beacon node. N2 is the slave beacon node of M1. $\mathrm{N}_{i}(i=1,2,3,4)$ is beacon node. $d_{i}(i=1,2,3)$ is the actual distance between beacon node to slave beacon node while $D_{i}(i=1,2,3)$ is the calculating distance between beacon node and slave beacon node. $c_{i}(i=1,2,3)$ is the calculated distance between blind node and beacon node [7].

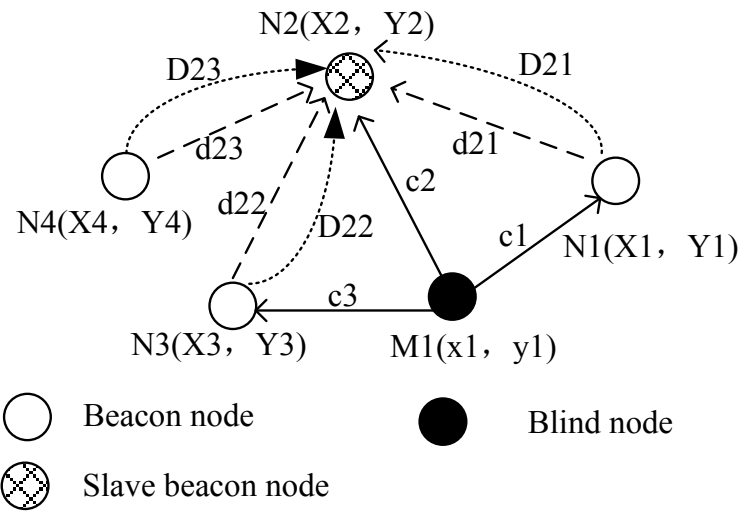

Fig. (1). Schematic diagram of a localization system.

\subsection{Initial Localization}

If the number of one-hop neighbor nodes around a blind node is more than three, the location of blind nodes can be calculated as follows [8].

(1) The slave node $\mathrm{N} 2$ receives communication packets from beacon nodes N1, N3, N4 and gets RSSI from N1, N3, N4. Since the location information of $\mathrm{N} i(i=1,2,3,4)$ is known, $d_{i}(i=1,2,3)$ is also known. Using equation (1) to calibrate parameters $P 0$ and $n$ for the certain environment. Binary linear regression was adopted to calibrate $P 0$ and $n$.

$R S S I=P 0-10 n \lg d$

(2) A proportional correction factor is defined by equation (2). Where, $k$ is the number of one-hop neighbor nodes. $d_{i}(i=1,2,3)$ is the actual distance between beacon node and slave beacon node, and can be calculated with the known location information of beacon node and slave beacon node. $D_{i}(i=1,2,3)$ is the calculated distance between beacon node and slave beacon node, and can be calculated by equation (1) and the parameters $P 0$ and $n$ has calibrated in step (1).
$\alpha=\sum_{i=1}^{k}\left(\frac{d_{i}-D_{i}}{d_{i}}\right)(k \geq 2)$

The proportional correction factor $\alpha$ is the average of relative measurement error for the distance between each beacon node and the slave node. It responses the degree of deviation of the distance measurement carried by RSSI and the corresponding relationship under the conditions of the different measurement regions.

(3) According to equation (1) and the parameters are calibrated in step (1) by calculating the distance $c_{i}(i=1,2,3)$.

(4) A distance corrections coefficient is defined by equation (3). Where, $\delta_{d}$ is given in equation (4). Equation (5) shows the calculation method of the radius of the inner circle and outer circle of a ring.

$$
\begin{aligned}
& \lambda_{i}=\exp \left(-\frac{c_{i}}{c_{i}+\alpha \delta_{d}}\right) \\
& \delta_{d}=\frac{1}{2} \sum_{i=1}^{2}\left(d_{i}-D_{i}\right) \\
& C N_{i}=c_{i} \pm \lambda_{i} \delta_{d}
\end{aligned}
$$

(5) The centroid of three rings overlapping region is regarded as the location of blind node. The rings are respectively round three beacon nodes. The results of equation (5) is the radius of the inner circle and outer circle of a ring. Rings localization principle can be shown in Fig. (2).

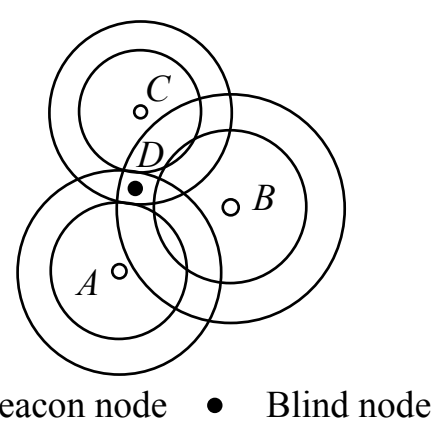

Fig. (2). Rings overlapping localization diagram with three one-hop neighbor nodes.

If the number of one-hop neighbor nodes around the blind node is less than three, the localization algorithm can be improved as follows $[9,10]$.

(1) Two beacon nodes are used to draw two rings by the method described above. Then, as shown in Fig. (3), take the centroid of the intersection regions $D$ and $D^{\prime}$ as the initial blind node estimated location.

(2) A two-hop beacon node $\mathrm{C}$ is used as the centre of a circle and the maximum communication distance $R$ is taken as 


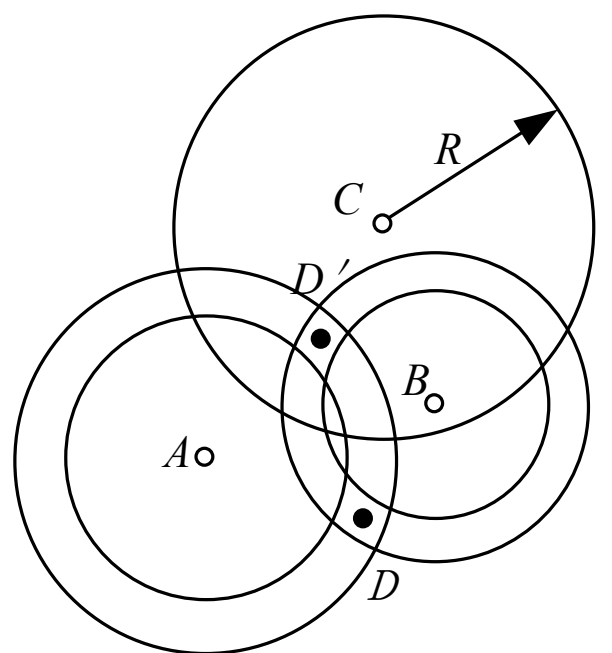

Fig. (3). Rings overlapping localization diagram with two one-hop and one two-hop neighbor nodes.

the radius to draw a circle named the communication circle. The initial blind node estimated location $D$ is outside of the communication circle and $D$ is the final blind node estimated location.

\subsection{Assisted Localization}

The omission node in the initial localization phase needs assisted localization as follows [11, 12].

(1) The slave beacon node is determined from the onehop neighbor beacon nodes. The slave beacon node receives communication packets, reads RSSI and calculates distance information. Then, binary linear regression is used to calibrate the parameters $P 0$ and $n$ for a certain environment. And then, the slave beacon node sends these parameters to other beacon nodes in its communication range.

(2) Blind node sends request to the neighbor beacon nodes. The assisted localization request contains the feedback times.

(3) The beacon nodes receive the assisted localization request and then give response. The transmit power will enhance accordance with feedback times.

(4) Blind node receives information and converts the assisted average RSSI into distance using equation (1). The parameters $P 0$ and $n$ has calibrated in step (1). The maximum likelihood estimation method is used in localization.

The maximum likelihood estimation method is as follows [13].

$$
\left(x_{i}, y_{i}\right)(i=1,2, \ldots \ldots, n) \text { is the coordinates of the } i \text { th bea- }
$$
con node. $M$ is the blind node and its coordinates are $\left(x_{m}\right.$, $\left.y_{m}\right) . r_{i}(i=1,2, \ldots \ldots, n)$ is the distance between the $i$ th beacon node and $M$. According to the equation of the distance between two points, equation (6) can be got.

$$
\left\{\begin{array}{c}
\left(x_{1}-x_{m}\right)^{2}+\left(y_{1}-y_{m}\right)^{2}=r_{1}^{2} \\
\left(x_{2}-x_{m}\right)^{2}+\left(y_{2}-y_{m}\right)^{2}=r_{2}^{2} \\
\vdots \\
\left(x_{n}-x_{m}\right)^{2}+\left(y_{n}-y_{m}\right)^{2}=r_{2}^{2}
\end{array}\right.
$$

Using the 1 to $n-1^{\text {th }}$ equations subtract the $n$th equation, equation (7) can be got.

$$
\begin{aligned}
& {\left[\begin{array}{cc}
2\left(x_{1}-x_{n}\right) & 2\left(y_{1}-y_{n}\right) \\
\vdots & \vdots \\
2\left(x_{n-1}-x_{n}\right) & 2\left(y_{n-11}-y_{n}\right)
\end{array}\right]\left[\begin{array}{l}
x_{m} \\
y_{m}
\end{array}\right]} \\
& =\left[\begin{array}{l}
x_{1}^{2}-x_{n}^{2}+y_{1}^{2}-y_{n}^{2}+r_{n}^{2}-r_{1}^{2} \\
x_{n-1}^{2}-x_{n}^{2}+y_{n-1}^{2}-y_{n}^{2}+r_{n}^{2}-r_{n-1}^{2}
\end{array}\right]
\end{aligned}
$$

Equation (7) can be solved by standard minimum mean square error estimation (MMSE). So, the coordinates of blind node $M\left(x_{m}, y_{m}\right)$ can be expressed as equation (8).

$X=\left(A^{T} A\right)^{-1} A^{T} b$

Where,

$$
\begin{aligned}
& A=\left[\begin{array}{cc}
2\left(x_{1}-x_{n}\right) & 2\left(y_{1}-y_{n}\right) \\
\vdots & \vdots \\
2\left(x_{n-1}-x_{n}\right) & 2\left(y_{n-11}-y_{n}\right)
\end{array}\right] \\
& X=\left[\begin{array}{l}
x_{m} \\
y_{m}
\end{array}\right] \\
& B=\left[\begin{array}{l}
x_{1}^{2}-x_{n}^{2}+y_{1}^{2}-y_{n}^{2}+r_{n}^{2}-r_{1}^{2} \\
x_{n-1}^{2}-x_{n}^{2}+y_{n-1}^{2}-y_{n}^{2}+r_{n}^{2}-r_{n-1}^{2}
\end{array}\right]
\end{aligned}
$$

\subsection{New Nodes Localization}

Due to the harsh environment, continuous work, attack and the unrenewable of nodes' energy, some nodes will be failure. New nodes need to join the network and get their location timely. In this way, the lifetime of the entire wireless sensor network can be extended. In order to get the location of new nodes, global calculation is not suitable while a region-independent calculation algorithm was adopted. New nodes collect the information of beacon nodes around them, and get their location by the above localization algorithm $[14,15]$.

\section{SIMULATIONS AND ANALYSIS}

\subsection{Relative Works}

In order to verify the correctness and validity of the proposed algorithm, simulation has done using MATLAB. MATLAB is one of the most popular mathematic software. 


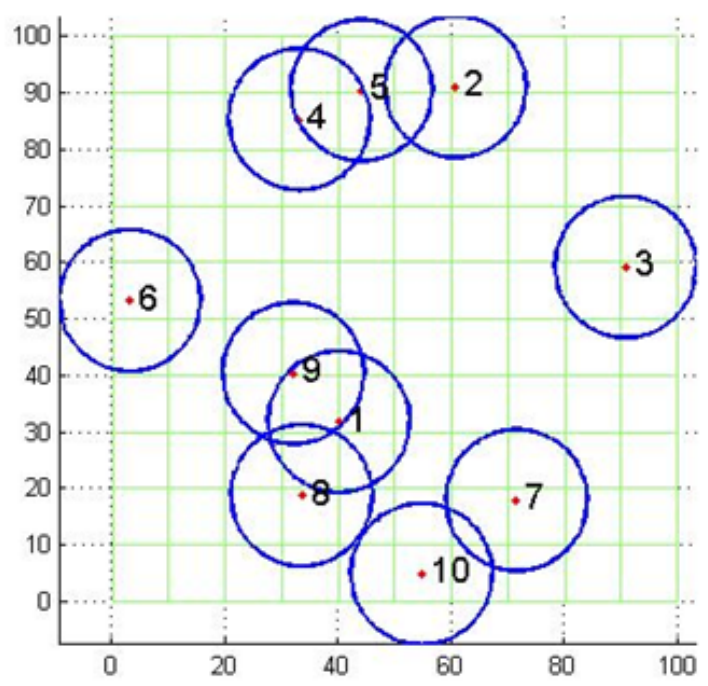

Fig. (4). 10 blind nodes in the simulation area.

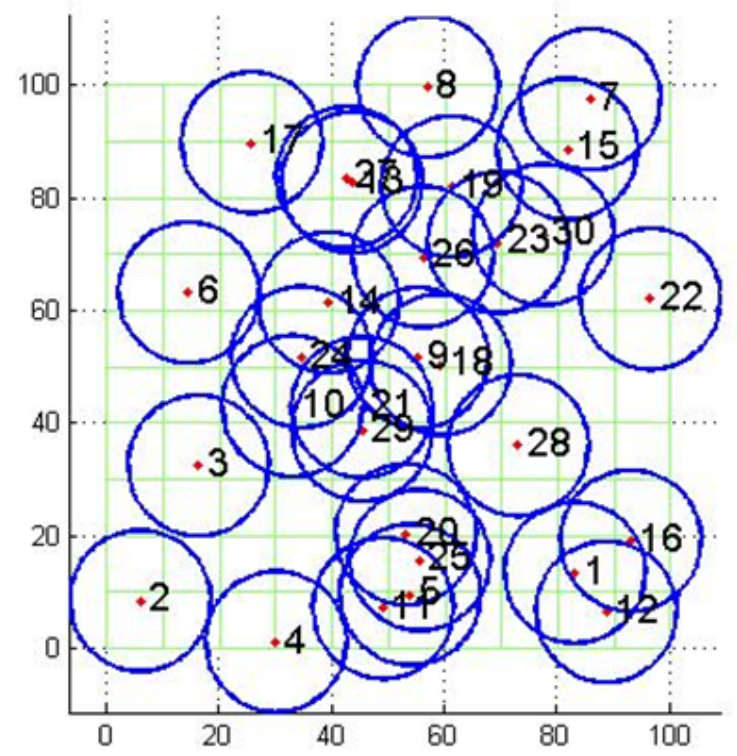

Fig. (5). 30 blind nodes in the simulation area.

It has been widely used for algorithm analysis, research and teaching. Using MATLAB, the maximum and minimum absolute error of $x$ coordinate, $y$ coordinate and location have been calculated. The comparison between the estimated location and the actual location of blind node has also simulated by MATLAB [16].

The number of beacon nodes is 121 and they are evenly distributed in an area of $100 r * 100 r . r$ is the unit length of the area and location error is measured by it. The amount of blind node is $5,10,15,20,25,30,35,40,45$ and 50 respectively, and the blind nodes are distributed at random. The communication distance of nodes is $12.5 r$. The side length of a grid is $10 r$.

Beacon nodes around a certain blind node are divided into two groups, one-hop beacon nodes and two-hop beacon nodes. Figs. (4-6) shows the situations of that, there are 10,
30 and 50 blind nodes in the simulation area respectively. Those beacon nodes within the communication range of a blind node are called one-hop beacon nodes. While other beacon nodes outside of the communication range but within twohop communication range are called two-hop beacon nodes.

\subsection{Simulation Results}

There are three kinds of evaluation criterion of localization algorithm performance. They are location error, $x$ localization error and $y$ localization error.

\subsubsection{Distance Error}

The location error is taken as the evaluation criterion of localization algorithm performance. The simulation results are shown in the Figs. (7-11). 


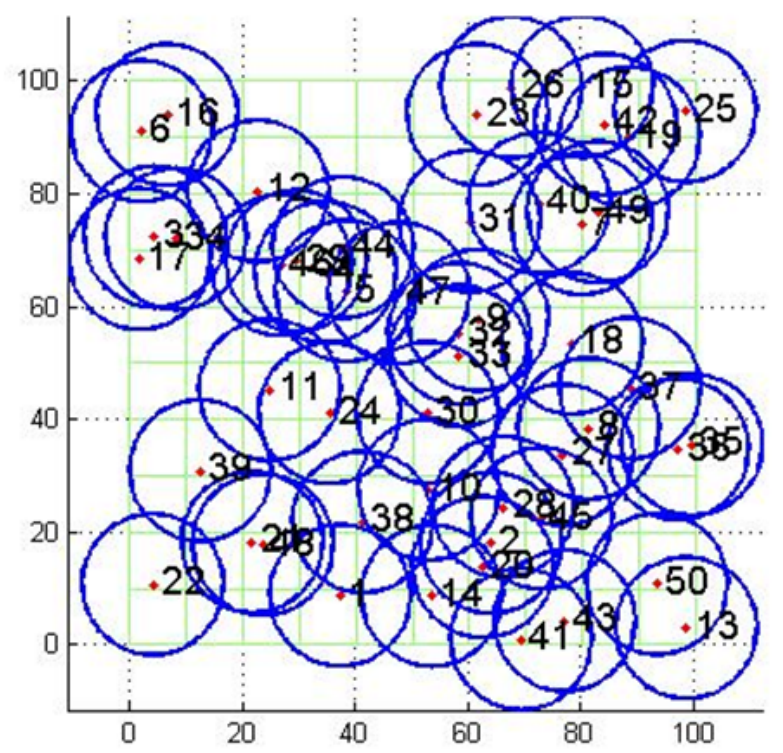

Fig. (6). 10 blind nodes in the simulation area.

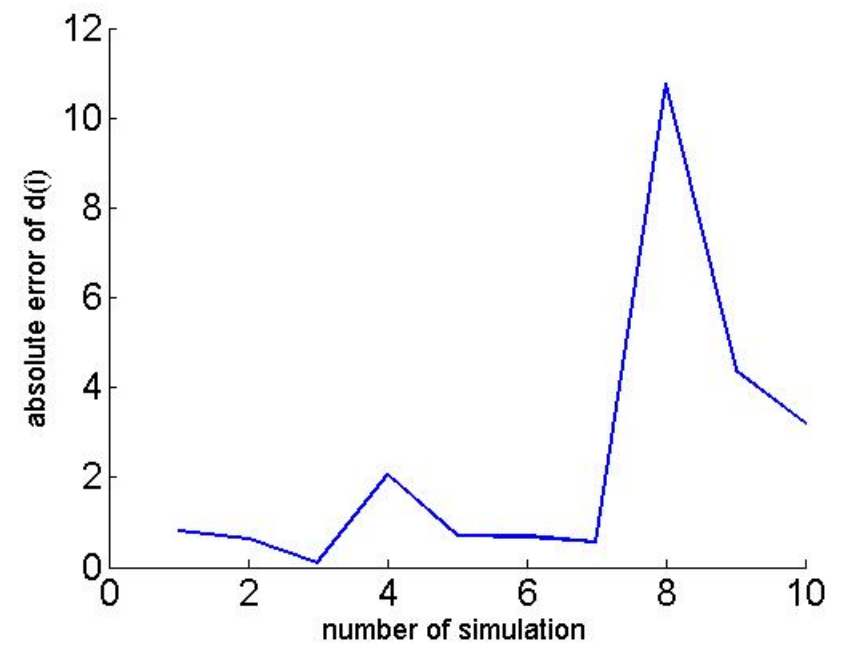

Fig. (7). Location error of 10 blind nodes.

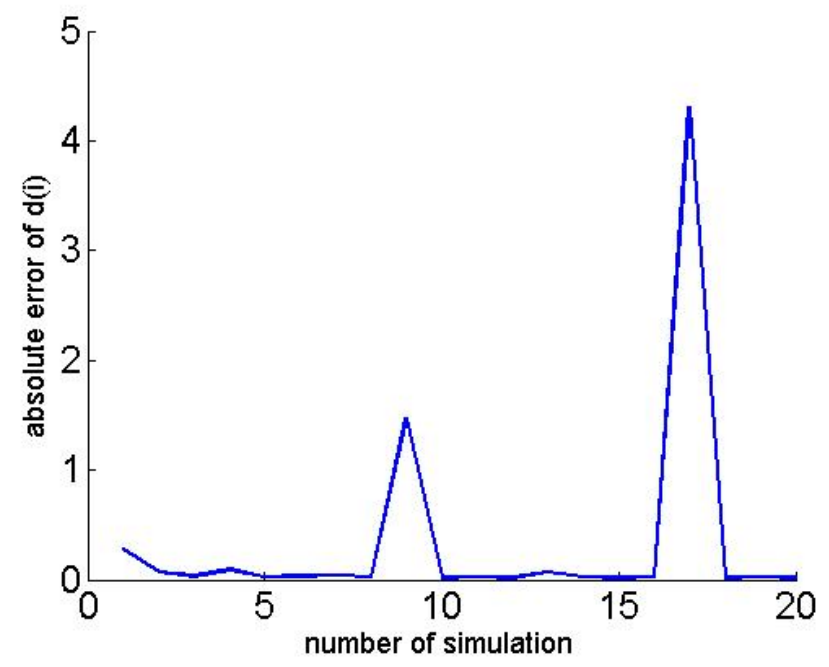

Fig. (8). Location error of 20 blind nodes. 


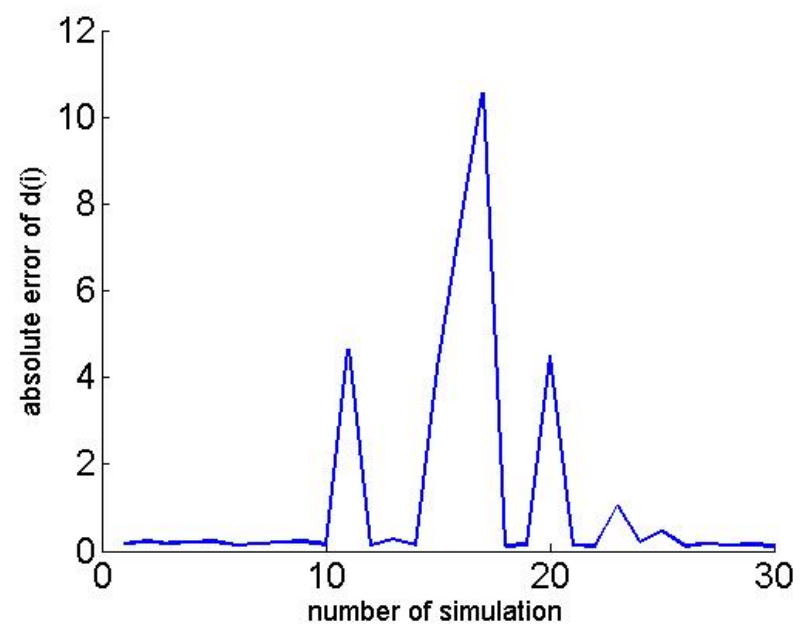

Fig. (9). Location error of 30 blind nodes.

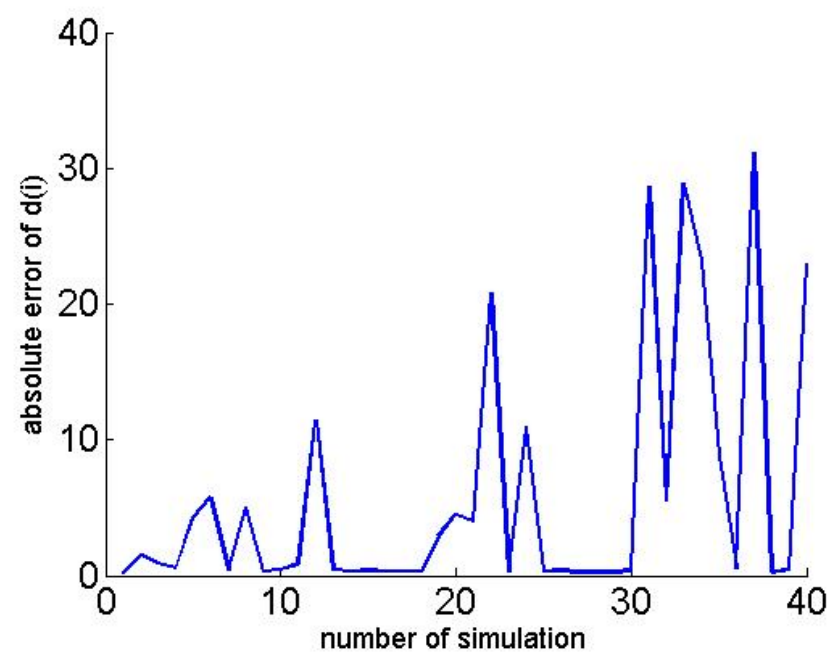

Fig. (10). Location error of 40 blind nodes.

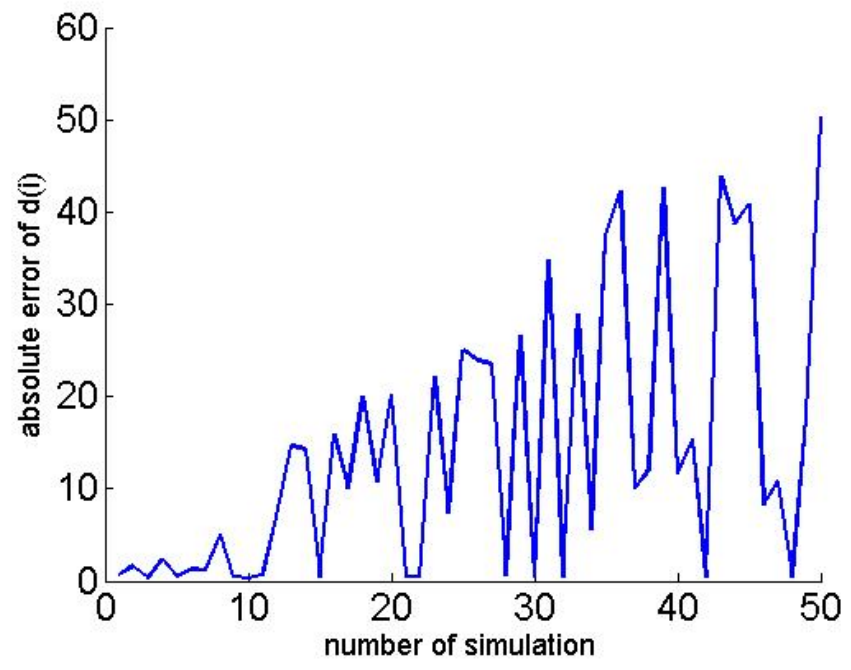

Fig. (11). Location error of 50 blind nodes. 


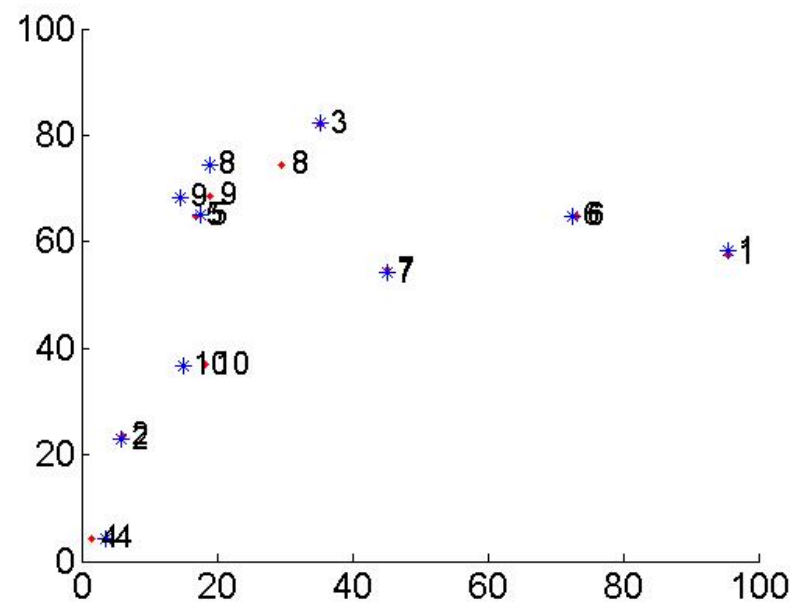

Fig. (12). Estimated location and actual location with 10 blind nodes.

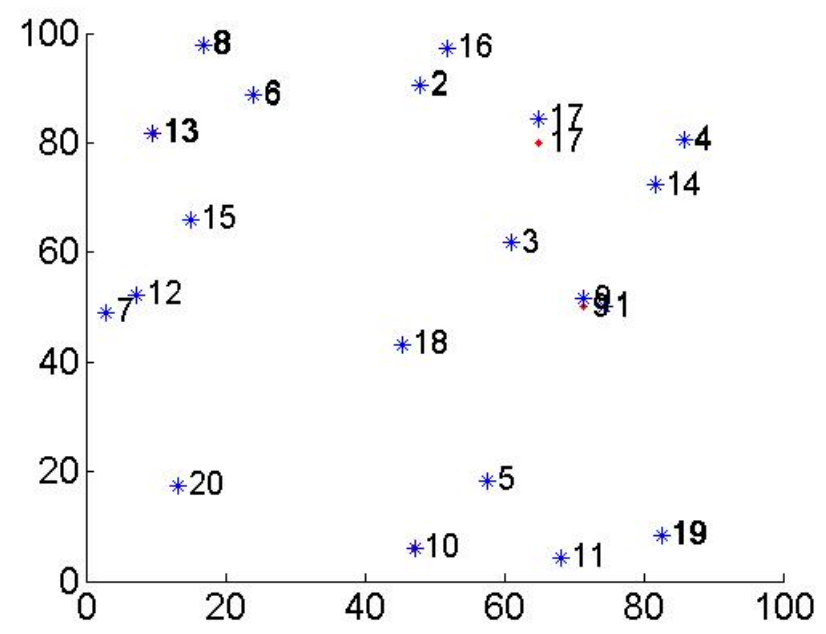

Fig. (13). Estimated location and actual location with 20 blind nodes.

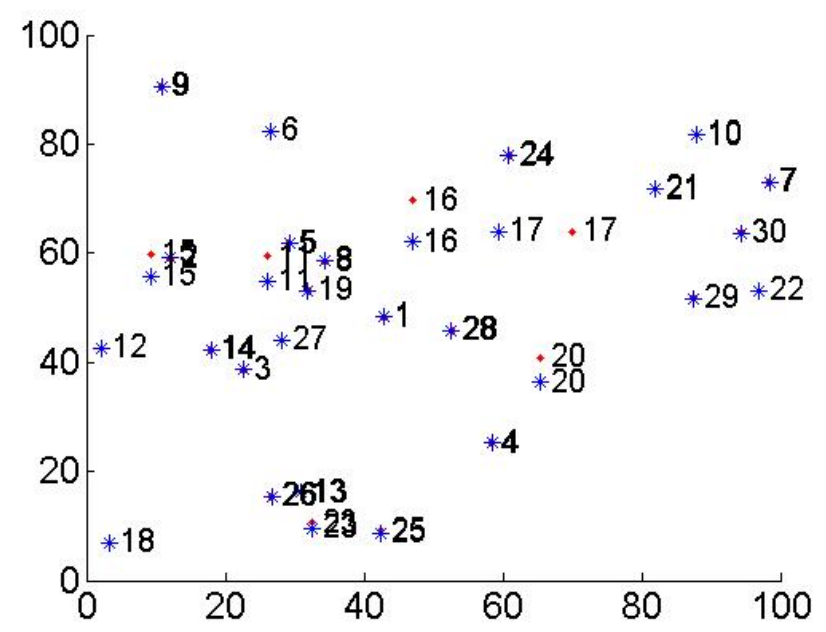

Fig. (14). Estimated location and actual location with 30 blind nodes. 


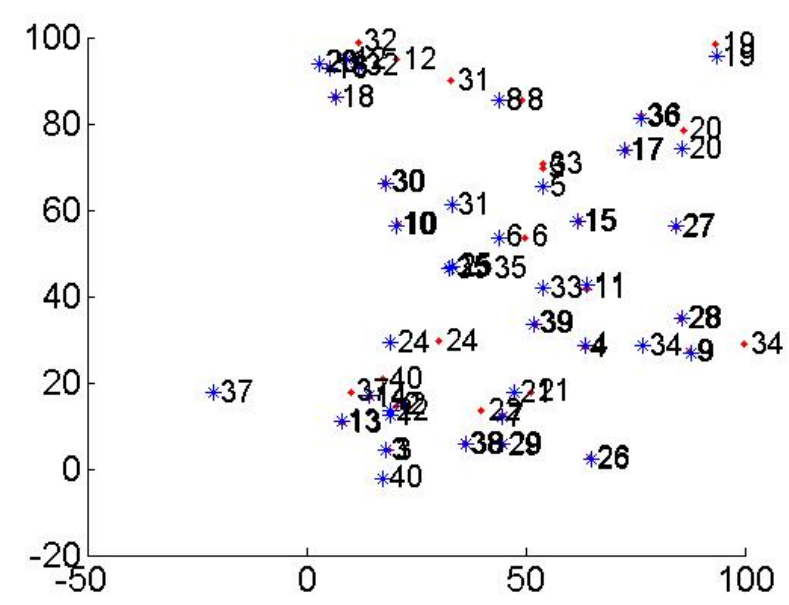

Fig. (15). Estimated location and actual location with 40 blind nodes.

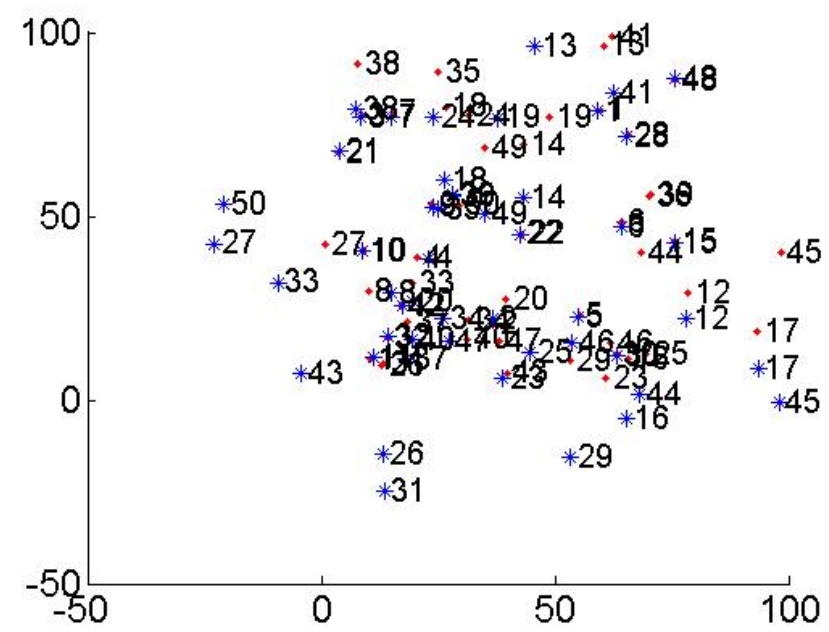

Fig. (16). Estimated location and actual location with 50 blind nodes.

Table 1. The location error of different blind nodes amount.

\begin{tabular}{|c|c|c|}
\hline Blind Node Amount & Error Greater than 0.5 $r$ (\%) & Error Greater than $r$ (\%) \\
\hline \hline 5 & 0 & 0 \\
\hline 10 & 0.2 & 0.1 \\
\hline 15 & 0.067 & 0.150 \\
\hline 20 & 0.2 & 0.040 \\
\hline 25 & 0.08 & 0.167 \\
\hline 30 & 0.233 & 0.143 \\
\hline 35 & 0.2 & 0.300 \\
\hline 40 & 0.475 & 0.022 \\
\hline 50 & 0.111 & 0.16 \\
\hline
\end{tabular}




\subsubsection{Comparison of Estimate Location and Actual Loca- tion}

Fig. (12-16) shows the comparison of the actual location and the estimated location.

\subsection{Analyses of Simulation Results}

With the increase of the number of blind node, the location error is shown in (Table $\mathbf{1}$ ).

Based on the data in (Table 1), there is no significant linear relationship between the location error and the number of blind node when beacon node number is certain. And, the actual location of blind node is also one of the factors that affect the location accuracy.

Generally speaking, the location error has a tendency to increase with the number of blind nodes. When there are less blind nodes in the simulation area, the localization error is smaller. While, when the blind nodes are distributed intensively, the location error is quite large. Even individual blind node localization would be failure. The right balance is somewhere in the middle, and that is the next phase of work to be done.

\section{CONCLUSION}

This paper presents a novel localization algorithm. The binary linear regression is used to calibrate the parameters of signal attenuation for a certain environment. Then, differential error estimate method is used to get the inner radius and outer radius of the rings which around beacon nodes and blind node respectively. At last, the centroid of the rings overlapping region is taken as the location of blind node. Different kinds of simulations are provided for the algorithm. The simulation results and analyses prove the correctness and validity of the localization algorithm.

\section{CONFLICT OF INTEREST}

The author confirms that this article content has no conflict of interest.

\section{ACKNOWLEDGEMENTS}

This work was financially supported by National Teacher Research Fund The $12^{\text {th }}$ Five-year Plan in The Ministry of Education (GBJ1421179), 2014 Key Project of Social Sci- ence League of Shan Xi Province (SSKLZDKT2014060) and The PhD Start-up Foundation of Taiyuan University of Science and Technology (W20122006).

\section{REFERENCES}

[1] W. T. Song, M. H. Tsai, "Multi-sensor wireless signal aggregation for environmental monitoring system via multi-bit data fusion", Appl. Math. Inf. Sci., vol. 5, pp. 589-603, 2011.

[2] Y. Qin, X. Zhang, "MSF-Based Speaker Automatic Emotional Recognition in Continuous Chinese Mandarin", Procedia Eng., vol. 15 , no. 11 , pp. 2229-2233, 2011.

[3] X. Liu, K. Chen, "A weighted localization algorithm using a hybrid of RSSI and TDOA range technologies", Comput. Digi. Eng., pp. 25-28, 2011

[4] Y. Qi, H. Fan, "Deregulation: reform trend of china's natural monopoly industries", China Indust. Eco., vol. 21, no. 4, pp. 62-72, 2009.

[5] J. Wang, S. Du, "Local area localization algorithm for underground tunnel based on WSN", Coal Mine Mach., pp. 89-91, 2010.

[6] W. Qu, L. Wang, Z. Liu, "Sensor localization with ring overlapping based on RSSI Using Three beacons", J. Northeastern Uni. Elect. Eng., pp. 16-19, 2012.

[7] Y. Qin, X. Zhang, "EEMD-sity(natural science)", J. Mech. Based Speaker Emot. Anal. Speech Sig., pp. 54-57, 2010.

[8] L. Mao, X. Lin, K. Shao, C. Ji, "Cooperative location algorithm in WSN based on RSSI and its application", Appl. Mech. Mater., vol. 121-126, pp. 815-819, 2012.

[9] S. Y. Moon and H. S. Seo, "Fuzzy Based Filtering Nodes Assigning Method for Sensor Networks", Appl. Math. Inf. Sci., vol. 6, pp. 285-289, 2012.

[10] J. Ho, H. Shih, B. Liao, J. Pan, "A reduce identical composite event transmission algorithm for wireless sensor networks", Appl. Math. Inf. Sci., vol. 6, no.2S, pp. 713S-719S, 2012.

[11] M. Xie, "EEORP: energy-efficient online reprogramming protocol for wireless sensor networks", Appl. Math. Inf. Sci., vol. 5, pp. 8996, 2011.

[12] Y. Qin, X. Zhang, "Fuzzy support vector machine-based emotional optimal algorithm in spoken chinese", $J$. Computat. Theoret. Nanosci., vol. 9, no. 10, pp. 1715-1719, 2012.

[13] Y. Qin, Y. Qi, "EEMD-based speaker automatic emotional recognition in chinese mandarin", Appl. Math. Inf. Sci., vol. 8, No. 2, pp. 617-624, 2014

[14] L. Mao, M. Xie, H. Jia, "SAS target recognition using improved fuzzy neural network", Sensors Transducers, vol. 155, no. 8, pp. 188-194, 2013

[15] N. Chayakom, R. Hubert, "Real-time 3D path and map estimation using a Multi-Camera system and a FastSLAM algorithm", Sensors Transducers, vol. 24, pp. 58-66, 2013.

[16] X. Niu, W. Meng, H. Cheng, "Research in the operational modal analysis of rotating machinery reducer", Sensors and Transducers, vol. 155 , no. 8 , pp. 255-261, 2013.

\footnotetext{
Received: September 22, 2014

Revised: November 03, 2014

Accepted: November 06, 2014

(C) Qin and Ying; Licensee Bentham Open.

This is an open access article licensed under the terms of the Creative Commons Attribution Non-Commercial License (http://creativecommons.org/licenses/by-nc/3.0/) which permits unrestricted, non-commercial use, distribution and reproduction in any medium, provided the work is properly cited.
} 